\title{
Low-temperature asymptotics of isotropic ferromagnetic chains at nonzero fields
}

\author{
N. Theodorakopoulos and N. C. Bacalis \\ Theoretical and Physical Chemistry Institute, National Hellenic Research Foundation, \\ Vas. Constantinou 48, GR - 11635 Athens, Greece
}

(Received 6 May 1996)

\begin{abstract}
We derive the leading-order asymptotics of classical one-dimensional isotropic ferromagnets near zero temperature in the context of two models with short-range interactions, namely the Heisenberg and the logarithmic chain. It is shown that the thermodynamics, in both cases, asymptotically reduce to the same differential equation, which also describes the azimuthally symmetric quantum motion of a rigid rotator in an external field. The result reflects the deep-rooted analogy between thermal and quantum fluctuations in classical $d$-dimensional and quantum $(d-1)$-dimensional systems, respectively. Explicit analytical expressions given in the limiting cases of high and low fields agree with the full numerical solution to within a few percent. [S0163-1829(97)06301-7]
\end{abstract}

Understanding the low-temperature thermal properties of one of the simplest categories of condensed-matter systems, the one-dimensional isotropic ferromagnets, has been a remarkably slow process. One of the major milestones was the numerical implementation of the thermodynamic Bethe ansatz for the $S=1 / 2$ Heisenberg case, ${ }^{1,2}$ which revealed that the zero-field susceptibility behaves in a classical-like fashion, i.e., $\chi_{0}(T) \propto 1 / T^{2}$.

The extension of the theory to nonzero fields revealed scaling behavior and a restricted universality with respect to the value of the spin: (i) the magnetization of the Heisenberg model was numerically shown to scale ${ }^{4}$ as $\rho \equiv h / T^{\sigma}$ (where $h$ denotes the magnetic field and $\sigma=2$ ); moreover, the scaling function was shown to be independent of the value of the spin. (ii) the same scaling property was shown to hold for the field-dependent part of the free energy (and thus for thermodynamics as a whole). [The demonstration of (ii) was made within the context of the related, soliton-bearing IshimoriHaldane-Faddeev ferromagnet (IHFF), ${ }^{5}$ and therefore suggests that (classical-like) scaling might be directly linked to the presence of classical-like solitons, i.e., solitons with high values of magnetization, which dominate field-dependent phenomena.]

At nonzero fields there are no analytical nonperturbative results for the thermodynamics of classical isotropic magnets. There is numerical evidence, ${ }^{4,5}$ that field-dependent thermodynamic properties (classical and quantum) near $T=0$ are characterized by a uniquely defined limiting ("scaling") function of $\rho$. The value of the scaling exponent $\sigma=2$, as well as some general properties of the scaling function have been derived ${ }^{5}$ on the basis of general thermodynamic arguments.

In order to obtain the exact form of the scaling function (as well as a complete description of low-temperature asymptotics of isotropic ferromagnets, including correlations) one must explicitly incorporate the scaling property " at the source," i.e., perform the $T \rightarrow 0$ limit on the constituent equation of classical statistical mechanics, i.e., the transferintegral (TI) equation. This recasts the problem in terms of the single state variable $\rho=h / T^{2}$; the limit of the (twoparameter) ferromagnetic TI equation will turn out to be a (single-parameter) Schrödinger-like equation, which describes the quantum mechanics of a rigid rotator. Moreover, it will be seen that the limit is independent of the details of the interaction, and manifests the deep-rooted formal analogy between thermal and quantum fluctuations in $d$ and $d-1$ dimensions, respectively. The derivation is as follows:

The model Hamiltonian is given by

$$
H / j=-\sum_{i} R\left(\hat{S}_{i} \cdot \hat{S}_{i+1}\right)-h \sum_{i} \hat{S}_{i}^{z}
$$

where the classical unit spin vectors $\left\{\hat{S}_{i}\right\}$ are placed in an external field $h$ in the $z$ direction, energies and fields are measured in units of the exchange constant $j$, and $R(x)=x-1$ (Heisenberg model, case $A$ in what follows), or $R(x)=-2 \ln [(1+x) / 2]$ (IHFF model, ${ }^{5}$ case $\left.B\right)$.

The original TI equations have the form

$$
\int_{-1}^{1} d x^{\prime} e^{\rho\left(x+x^{\prime}\right) / n} K_{0}\left(x, x^{\prime} ; n\right) \psi_{l}\left(x^{\prime}\right)=\lambda_{l} \psi_{l}(x),
$$

where

$$
\begin{aligned}
& K_{0}\left(x, x^{\prime} ; n\right) \\
& =\left\{\begin{array}{cc}
e^{n x x^{\prime} / 2} I_{0}\left(n \sqrt{1-x^{2}} \sqrt{1-x^{\prime 2}} / 2\right) \quad(A)(\text { Ref. 6) } \\
\left|\frac{x+x^{\prime}}{2}\right| P_{n}\left(\frac{1+x x^{\prime}}{\left|x+x^{\prime}\right|}\right) \quad(B)(\text { Ref. 5), }
\end{array}\right.
\end{aligned}
$$

where $n=2 T^{-1}$, and $I_{0}, P_{n}$ denote Bessel and Legendre functions, respectively.

Asymptotic expansions. We are interested in the limiting form of cases $A$ and $B$ in Eq. (3) in the limit $n \rightarrow \infty$. In this limit, the asymptotic expansion $I_{0}(z) \sim 1 / \sqrt{2 \pi z} e^{z}\left[1+1 /(8 z)+\mathcal{O}\left(1 / z^{2}\right)\right]$ (Ref. 7) can be used. We have not been able to find an analogous asymptotic expansion for the Legendre function [valid for large values of (noninteger) $n$, and including the relevant first-order correction] in the standard tables. Our derivation in case $B$ rests on the validity of the asymptotic expansion for the Legendre function, 


$$
\begin{aligned}
P_{n}(z) \sim & \frac{1}{2 \pi n}\left(z+\sqrt{z^{2}-1}\right)^{n+1 / 2}\left\{1-\frac{1}{4 n}\left(1-\frac{1}{2} \frac{z}{\sqrt{z^{2}-1}}\right)\right. \\
& \left.+\mathcal{O}\left(\frac{1}{n^{2}}\right)\right\}, \quad|z|>1
\end{aligned}
$$

which we have derived for integer values of $n$. (There is no such restriction in case $A$ ). The leading term is in agreement with published results; ${ }^{8}$ so are the values of $\lim _{z \rightarrow \infty}\left\{z^{-n} P_{n}(z)\right\}$.
Transformation of Eq. (2) to the original polar coordinates $x=\cos \theta, x^{\prime}=\cos \theta^{\prime}$ leads to

$$
\begin{aligned}
& \frac{1}{\sqrt{\pi n}} \int_{0}^{\pi} d \theta^{\prime} e^{\rho\left(\cos \theta+\cos \theta^{\prime}\right) / n}\left(\frac{\sin \theta^{\prime}}{\sin \theta}\right)^{1 / 2} \Phi\left(\theta^{\prime}, \theta\right) \psi_{l}\left(\theta^{\prime}\right) \\
& \quad=\lambda_{l} \psi_{l}(\theta)
\end{aligned}
$$

where

$$
\Phi\left(\theta^{\prime}, \theta\right)=\left\{\begin{array}{c}
{\left[1+\frac{1}{4 n \sin \theta \sin \theta^{\prime}}\right] e^{-n \sin ^{2}\left[\left(\theta^{\prime}-\theta\right) / 2\right]} \quad(A)} \\
{\left[1-\frac{1}{4 n}\left(1-\frac{1+\cos \theta \cos \theta^{\prime}}{\sin \theta \sin \theta^{\prime}}\right)\right] \cos ^{2 n+1}\left(\frac{\theta^{\prime}-\theta}{2}\right)}
\end{array}\right.
$$

The presence of $n$ as an exponent in the kernel (6) means that, in the limit $n \rightarrow \infty$, only a very narrow region of typical width $1 / \sqrt{n}$ around $\theta^{\prime}=\theta$ can contribute to the integral. This allows us to make a second-order Taylor expansion of $\psi\left(\theta^{\prime}\right)$ and the other, slowly varying parts of the kernel, and evaluate the resulting Gaussian integrals by extending the limits to $\pm \infty$. Keeping terms of order $1 / n$ everywhere, we arrive at

$$
\psi_{l}^{\prime \prime}(\theta)+\cot \theta \psi_{l}^{\prime}(\theta)+2\left[\rho \cos \theta+\mu_{l}\right] \psi_{l}(\theta)=0,
$$

where $\mu_{l}=\lim _{n \rightarrow \infty}\left[(n / 2)\left(1-n \lambda_{l} / 2\right)\right]-c$ and $c$ equals 0 $(A)$, or $1 / 2(B)$. This completes our derivation of Eq. (7) as the (common) limit of Eq. (2) as $n \rightarrow \infty$. In physical terms, the ground state of the (zero-dimensional) quantum rotator, described by Eq. (7) is directly linked to the highest eigenstate of Eq. (2), i.e., to the field-dependent part of thermal fluctuations of the classical (and by virtue of Refs. 4,5 the quantum) isotropic one-dimensional chain near $T=0$. The precise content of the asymptotic mapping of Eq. (2) to Eq. (7) is that the field-dependent part of the free energy $\delta f$ satisfies

$$
T^{-2} \delta f(\rho) \equiv-T^{-1} \ln \left\{\frac{\lambda_{0}(\rho)}{\lambda_{0}(0)}\right\} \sim \mu_{0}(\rho),
$$

to leading order in $1 / n$, for both cases $A$ and $B$.

A technical comment is in order here, regarding the (different) values of the constant $c$ in cases $A$ and $B$. The values are correct, in the sense that they reproduce the lowtemperature expansions of $\lambda_{0}(0)$, obtained from the exact TI values, including $\mathcal{O}\left(T^{2}\right)$ terms; in particular, the absence of powers of $T$ (beyond the first) in the energy of the Heisenberg model $^{3}$ is confirmed. The different values of $c$ also imply that the field-independent properties are sensitive to the details of the interaction $R(x)$ and one should be careful in the use of $B$ to describe $A$ beyond leading order. It should be further noted that field-independent properties are sensitive to the value of the spin; this "dichotomy" between field-dependent and field-independent aspects of statistical mechanics can be best understood within the soliton picture. $^{9,5}$

In the limit of large $\rho$, we find that the two lowest states of Eq. (7) are given by

$$
\begin{aligned}
& \mu_{0}(\rho) \sim-\rho+\sqrt{\rho}-\frac{1}{4}-\frac{1}{64 \sqrt{\rho}}, \\
& \mu_{1}(\rho) \sim-\rho+3 \sqrt{\rho}-\frac{3}{4}-\frac{9}{64 \sqrt{\rho}} .
\end{aligned}
$$

The first two terms in the above equations have been reported by von Meyenn. ${ }^{10}$ The leading-order term comes from the complete orientation of the rotator along the $z$ axis. The next term describes the low-amplitude oscillations of the hin-

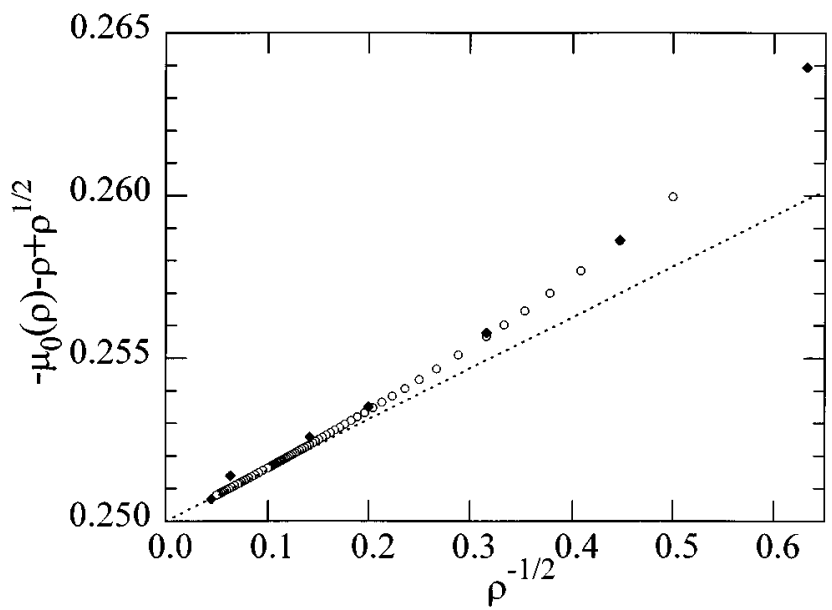

FIG. 1. Numerical results for (minus) the lowest eigenvalue of Eq. (7) vs $1 / \sqrt{\rho}$ for large $\rho$ (squares). Note that we have subtracted away the two leading terms in Eq. (9). The data of Ref. 10 are also shown (solid diamonds). The dotted line represents the known terms of the asymptotic expansion Eq. (9). 


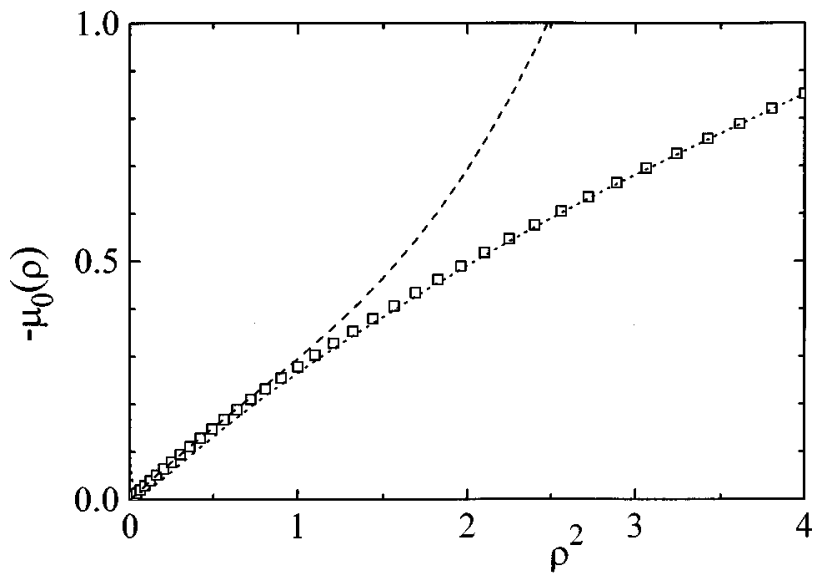

FIG. 2. (Minus) the lowest eigenvalue of Eq. (7) vs $\rho^{2}$ (squares). Also shown are (i) the (first three terms of the) power expansion (12) (dashed line) and the extrapolation of a sixth-order asymptotic expansion obtained by polynomial regression from the numerical data of Fig. 1.

dered rotator around $\theta=0$ for high values of the field. We have derived the next two terms using second-order perturbation theory around the exact hindered rotator states. The coefficient $1 / 4$ has also been obtained numerically in Ref. 5 as $G_{\infty} \equiv \lim _{\rho \rightarrow \infty}\left[-\mu_{0}(\rho)+2 \rho \mu_{0}^{\prime}(\rho)+\rho\right]$ and is consistent with the numerical results of Ref. 10, where a value of 0.02525 is reported at $\rho=50$ (note the slightly different notations; $\mu_{0}(\rho)$ corresponds to $-g(x)$ of Ref. 5).

We have performed a numerical evaluation of $\mu_{0}(\rho)$ and its derivatives and reproduced the thermodynamics presented as "scaling limit" in Ref. 5 within numerical accuracy. In Fig. 1 we plot $\mu_{0}(\rho)$ vs $1 / \sqrt{\rho}$.

At low values of $\rho$, it is possible to exploit the expansion of $\psi_{0}(\theta)$ in terms of Legendre polynomials; the expansion coefficients $\left\{A_{j}\right\}$ satisfy the recurrence relationship

$$
2 \rho \frac{j}{2 j-1} A_{j-1}-[j(j+1)+2 \mu] A_{j}+2 \rho \frac{j+1}{2 j+3} A_{j+1}=0 .
$$

Truncating after a fixed, finite number of terms produces a matrix eigenvalue problem; successive iteration can be used analytically, to produce a systematic expansion for the lowest eigenvalue $\mu_{0}(\rho)$ (alternatively, truncating after a large number of terms, yields a direct numerical result). We give the first three terms:

$$
-\mu_{0}(\rho)=\frac{1}{3} \rho^{2}-\frac{11}{3 \cdot 45} \rho^{4}+\frac{8 \cdot 47}{3 \cdot 45 \cdot 63} \rho^{6}+\ldots .
$$

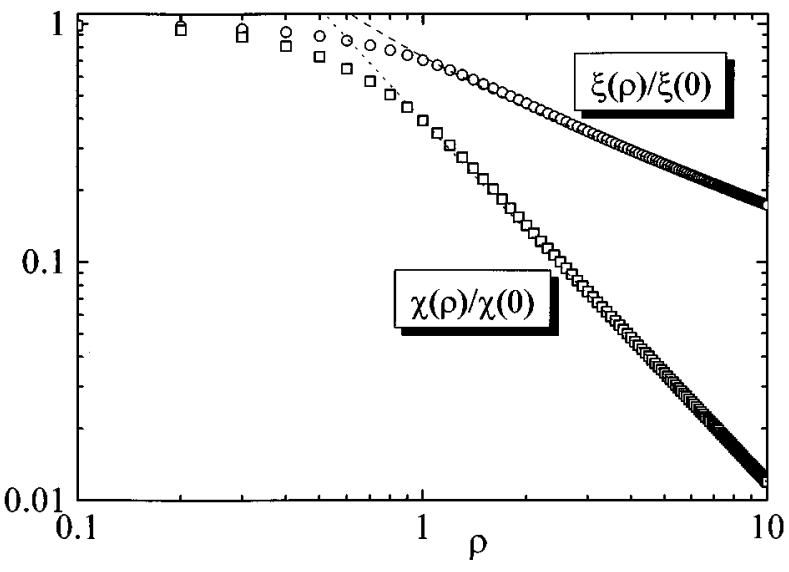

FIG. 3. The susceptibility (squares) and correlation length (circles) vs $\rho$. Both quantities are divided by their zero-field values. Also shown are our results in terms of the asymptotic expansions $\chi(\rho) / \chi(0) \sim 3 / 8 \rho^{-3 / 2}+9 / 512 \rho^{-5 / 2} \quad$ (dotted line), and $\xi(\rho) / \xi(0) \sim 1 /\left[2 \rho^{1 / 2}-1 / 2-1 / 8 \rho^{-1 / 2}\right]$ (dashed line).

The first term in Eq. (12) reproduces Fisher's zero-field susceptibility; the second term has been recently derived in a nonlinear susceptibility calculation. ${ }^{4}$ In Fig. 2 we plot the approximation (12), along with the numerical results and the extrapolation from the high- $\rho$ asymptotics.

We conclude our account of the asymptotic thermodynamics of Eq. (1) with the results for the susceptibility $\chi$ and correlation length $\xi$. In Fig. 3 we plot $T^{2} \chi=-\mu_{0}^{\prime \prime}(\rho)$ and $T \xi=1 /\left(\mu_{1}-\mu_{0}\right)$ vs $\rho$. Asymptotic expansions to next-toleading order [readily obtainable from Eqs. (9) and (10)] are also plotted (cf. caption for details). The dependence of the correlation length as $1 / \sqrt{h}$ in the high-field regime allows us to look at scaling behavior as the competition of two physical length scales, $\xi_{0} \propto 1 / T$ (Ref. 3) and $\xi_{\infty} \propto 1 / \sqrt{h}$. At any finite value of the field, the system interpolates between the two regimes; the field dependence of thermodynamic functions thus occurs via the dimensionless ratio $\xi_{0} / \xi_{\infty}$ $\propto \sqrt{h} / T[\equiv \sqrt{\rho}]$.

In summary, we have shown that, in the asymptotic limit $T \rightarrow 0$, the transfer integral equations describing the classical statistical mechanics of two exemplary isotropic ferromagnetic chains, reduce to the eigenvalue equation of the quantum rotator, where the scaling variable $\rho \equiv h / T^{2}$ is the only parameter. As a consequence, thermal fluctuations in onedimensional isotropic systems correspond, near absolute zero, to the ground state of the quantum rotator.
${ }^{1}$ P. Schlottmann, Phys. Rev. Lett. 54, 2131 (1985); Phys. Rev. B 33, 4880 (1986).

${ }^{2}$ M. Takahashi and M. Yamada, J. Phys. Soc. Jpn. 54, 2808 (1985).

${ }^{3}$ M.E. Fisher, Am. J. Phys. 32, 343 (1964).
${ }^{4}$ H. Nakamura and M. Takahashi, J. Phys. Soc. Jpn. 63, 2563 (1994).

${ }^{5}$ N. Theodorakopoulos, N.C. Bacalis, and Z. Xiong, Phys. Rev. B 54, 4033 (1996).

${ }^{6}$ M. Blume, P. Heller, and N.A. Lurie, Phys. Rev. B 11, 
4483 (1975).

${ }^{5}$ M. Abramowitz and I. Stegun, Handbook of Mathematical Functions (Dover, New York, 1965), p. 377.

${ }^{6}$ W. Magnus, F. Oberhettinger, and R.P. Soni, Formulas and Theorems for the Special Functions of Mathematical Physics
(Springer, Berlin, 1966), p. 235.

${ }^{7}$ N. Theodorakopoulos and N.C. Bacalis, Phys. Rev. Lett. 67, 3018 (1991).

${ }^{8}$ K. von Meyenn, Z. Phys. 231, 154 (1970). 